\title{
Propiedades mecanicas y tribológicas del material antifricción Al-Ni-grafito obtenido mediante extrusion en caliente
}

\author{
L.E.G.CAMBRONERO, R.ROSELL, J.M.RUIZ-ROMÁN, JM RUIZ-PRIETO \\ Departamento de Ingeniería de Materiales. E.T.S.I. Minas, 28003 Madrid
}

\begin{abstract}
El objeto de este trabajo es la obtención y caracterización de materiales compuestos (MMC's) para la fabricación de cojinetes para fricción en seco. Así se incorpora a una matriz de aluminio, partículas de grafito para actuar como lubricante si bien en forma de grafito recubierto de níquel para disminuir la perdida de resistencia que presentan estos materiales. La fabricación de estos materiales se realizó mediante compactación de las mezclas de polvos en matriz y su extrusión en caliente. Los materiales obtenidos presentaron una buena distribución del grafito en la matriz de aluminio, con densidades entre $3.11 \mathrm{~g} / \mathrm{cm}^{3}$ y $3.75 \mathrm{~g} / \mathrm{cm}^{3}$ según el contenido de níquel/grafito. La dureza y la resistencia al aplastamiento radial disminuyen al aumentar el contenido en grafito. No obstante, un mayor contenido en grafito si mejora las propiedades a fricción del material, disminuyendo el desgaste ocasionado y reduciendo el coeficiente de fricción. El tratamiento térmico de difusión de los materiales extruidos mejora sus propiedades de fricción si bien disminuye su resistencia al aplastamiento radial.
\end{abstract}

Palabras clave: Cojinetes, aluminio-grafito, extrusión de polvos, desgaste

Mechanical and tribological properties of hot extruded al-ni-graphite antifriction material

Manufacturing and characterization of bearing materials is the object of the present work. Thus, graphite particles are added to an aluminium matrix as lubricant. It was added as nickel coated graphite in order to avoid a strength decreasing. Mixing of powders following by cold compaction and hot extrusion was the manufacturing way. Extruded materials showed good graphite distribution into the aluminium matrix, with densities between 3.11 to $3.75 \mathrm{~g} / \mathrm{cm}^{3}$ as a function of the nickel/graphite content. Hardness and radial crushing strength decrease when graphite content is increased. On the contrary, friction properties are improved with the graphite content because it decreases friction coefficient and wear. Diffusion heat treatment on extruded materials improves furthermore these friction properties but it decreases the radial crushing strength.

Keywords: Bearings, Aluminium-Graphite, powder extrusion, wear

\section{INTRODUCCION}

En los metales de alta conductividad térmica destinados para aplicaciones de fricción como los cojinetes de deslizamiento, una forma de incrementar su capacidad de carga y reducir su desgaste es mediante la reducción del coeficiente de fricción con la adición de lubricantes que para bajas velocidades $(<0.5 \mathrm{~m} / \mathrm{s})$ se puede realizar con la incorporación de grafito. Sin embargo las mezclas de polvos metal-grafito presentan una mala sinterabilidad. Como alternativa se ha planteado el uso de polvos de grafito recubierto de níquel que han permitido resolver la baja sinterabilidad de los bronces con grafito [1-4], habiéndose incorporado a estos materiales mediante la mezcla de polvos, consolidación por compactación, sinterización y tratamiento térmico de maduración, dado que la difusión del níquel en la matriz $\mathrm{Cu}$-Sn permite obtener una fase Ni-Sn que endurece al material. Estos bronces al níquel con grafito plantean unas adecuadas propiedades para su empleo en el campo de los cojinetes para fricción en seco. Es por todo ello que en el presente trabajo se plantea obtener materiales compuestos de matriz de aluminio con grafito, pero en lugar de mediante fusión o colada que también incorporan estos polvos de níquel/grafito (Ni/Gr) en su procesado [5,6], empleando las técnicas de la pulvimetalurgia. Así la fabricación de estos materiales se plantea mediante las etapas de mezcla de polvos, compactación y extrusión en caliente. En los materiales obtenidos se determinaran la densidad, resistencia al aplastamiento radial, dureza, coeficiente de fricción y microestructura.

\section{PROCEDIMIENTO EXPERIMENTAL}

\subsection{Materias primas.}

Se prepararon tres mezclas de polvos de composiciones (en peso) $\mathrm{Al}+30 \% \mathrm{Ni} / \mathrm{Gr}, \mathrm{Al}+40 \% \mathrm{Ni} / \mathrm{Gr}$ y $\mathrm{Al}+60 \% \mathrm{Ni} / \mathrm{Gr}$, mediante mezclado manual. Como polvos de partida se emplearon polvos de aluminio (tamaño de partícula $<400 \mu \mathrm{m}$, velocidad de flujo: $85 \mathrm{~s} / 50 \mathrm{~g}$, 99,5\% Al, 2,66 $\mathrm{g} / \mathrm{cm}^{3}$ ) y polvos de grafito recubierto de níquel (tamaño de partícula $<150 \mu \mathrm{m}$, velocidad de flujo: $\left.35 \mathrm{~s} / 50 \mathrm{~g}, 75 \% \mathrm{Ni}, 4,75 \mathrm{~g} / \mathrm{cm}^{3}\right)$ junto con un $0,4 \%$ de lubricante en polvo.

\subsection{Compactación y extrusión en caliente}

Entre los posibles métodos de conformación y consolidación de los polvos obtenidos, se aplicó la extrusión directa en caliente de los compactos en verde, previa lubricación de los mismos con grafito [7]. Estos compactos de forma cilíndrica ( $25 \mathrm{~mm}$ de diámetro) se obtuvieron mediante compactación de las mezclas de polvos a una presión de 300MPa. La extrusión en caliente se realiza a $500^{\circ} \mathrm{C}$ a una velocidad de $0.1 \mathrm{~m} / \mathrm{s}$ y con una relación de extrusión de 5:2, obteniéndose una barra para cada material $(\mathrm{Al}+30 \% \mathrm{Ni} / \mathrm{Gr}, \mathrm{Al}+40 \% \mathrm{Ni} / \mathrm{Gr}$ y $\mathrm{Al}+60 \% \mathrm{Ni} / \mathrm{Gr})$ de $10 \mathrm{~mm}$ de diámetro. Mediante mecanización de cada barra se obtuvieron probetas para dilatometría $(20 \mathrm{~mm} \times 3 \mathrm{~mm} \times 3 \mathrm{~mm})$, análisis microestructural y ensayos mecánicos.

\subsection{Análisis dilatométrico y Tratamiento térmico.}

Para determinar el comportamiento de los materiales extruidos con la temperatura y su coeficiente de dilatación térmica, se realizaron diversos análisis dilatométricos en un dilatómetro Bahr a temperaturas de $400^{\circ} \mathrm{C}, 525^{\circ} \mathrm{C}$ y $575^{\circ} \mathrm{C}$, con duraciones de $0-4 \mathrm{~h}$, y velocidades de calentamiento de $5^{\circ} \mathrm{C} / \mathrm{min}$ en vacío. Como resultado del mismo, las condiciones seleccionadas para el tratamiento térmico de las probetas fueron: $525^{\circ} \mathrm{C} / 4 \mathrm{~h}$ en vacío. 


\subsection{Propiedades físicas, mecánicas y tribológicas.}

La densidad en verde se obtuvo según UNE-EN-ISO 3927, mientras que la densidad del material extruido se realizo mediante un picnómetro de gas ACCUPYC1330 a $20^{\circ} \mathrm{C} /$ Argón. Se determinaron la resistencia al aplastamiento radial según la norma EN ISO 2739 sobre casquillos mecanizados de 5,5mm de diámetro interior y la dureza $\mathrm{HRH}$ en todas las piezas, tanto casquillos como cilindros obtenidas de la barra extruida. El comportamiento tribológico de estos materiales se realizó mediante un equipo "pin-on-disk" , con una bola de acero al cromo como "pin" con cargas de 2 y $5 \mathrm{~N}$ a velocidades de $8 \mathrm{~cm} / \mathrm{s}$, durante $214 \mathrm{~m}$, en probetas pulidas $(\mathrm{Ra}<0.5 \mu \mathrm{m})$.

El estudio microestructural mediante microscopia óptica y electrónica de barrido se complemento con la determinación de la microdureza empleando una carga de $50 \mathrm{~g}$ durante $12 \mathrm{~s}$. El análisis de la fractura en los casquillos ensayados a rotura se completa con el ensayo a flexión de las probetas sometidas a dilatometría y con una cara pulida.

\section{RESULTADOS}

3.1 Compresibilidad y Extrusionabilidad de las mezclas de polvos.

Las composiciones de $\mathrm{Al}+30 \% \mathrm{Ni} / \mathrm{Gr}$ y $\mathrm{Al}+40 \% \mathrm{Ni} / \mathrm{Gr}$ mostraron una adecuada conformabilidad mediante compactación en matriz y extrusión en caliente. Por el contrario, la superficie de las barras extruidas con la mezcla $\mathrm{Al}+60 \% \mathrm{Ni} / \mathrm{Gr}$ mostraron importantes grietas y por ello no se caracterizaron estos materiales. Para os materiales $\mathrm{Al}+30 \% \mathrm{Ni} / \mathrm{Gr}$ y $\mathrm{Al}+40 \% \mathrm{Ni} / \mathrm{Gr}$ se alcanzaron densidades en verde de 3,02-3,03 g/ $\mathrm{cm}^{3}$ y tras extrusión de $3.11 \mathrm{~g} / \mathrm{cm}^{3}$ y $3.75 \mathrm{~g} / \mathrm{cm}^{3}$ respectivamente.

\subsection{Microestructura de los materiales extruidos.}

Se ha alcanzado una adecuada distribución de las partículas de $\mathrm{Ni} / \mathrm{Gr}$ en la matriz de aluminio (Figura 1), si bien dada su morfología plana, estas partículas se alinean en la dirección de extrusión. No se observan en ningún material una zona de difusión entre el níquel y la matriz, ni la presencia de precipitados o porosidad en la misma.

\subsection{Materiales tratados térmicamente.}

El comportamiento dilatométrico de los materiales mostró una variación uniforme de su dilatación durante el calentamiento o enfriamiento. Los coeficientes de dilatación lineal alcanzados fueron de $2510^{-6} \mathrm{~K}^{-1}$ para el $\mathrm{Al}+30 \% \mathrm{Ni} / \mathrm{Gr}$ y de $2010^{-6} \mathrm{~K}^{-1}$ para el $\mathrm{Al}+40 \% \mathrm{Ni} / \mathrm{Gr}$. Durante la permanencia a las distintas temperaturas se observó una contracción progresiva del $0.03 \%$ para $400^{\circ} \mathrm{C}$, hasta el $0.2 \%$ para $575^{\circ} \mathrm{C}$ en ambos materiales.

Desde el punto de vista microestructural, se observa tras el tratamiento térmico a temperaturas de $525^{\circ} \mathrm{C}$ o superiores, una transformación de las áreas de níquel a altas temperaturas. Así la difusión del aluminio conduce probablemente al ínter metálico $\mathrm{Ni}_{3} \mathrm{Al}$, si bien en algunas áreas no se alcanza la total difusión del níquel (Figura 2A) y se puede observar una transición desde la matriz de aluminio a las zonas de $\mathrm{Ni}-\mathrm{Al}$, al $\mathrm{Ni}$ y al área de grafito. Así mismo esta difusión conduce a
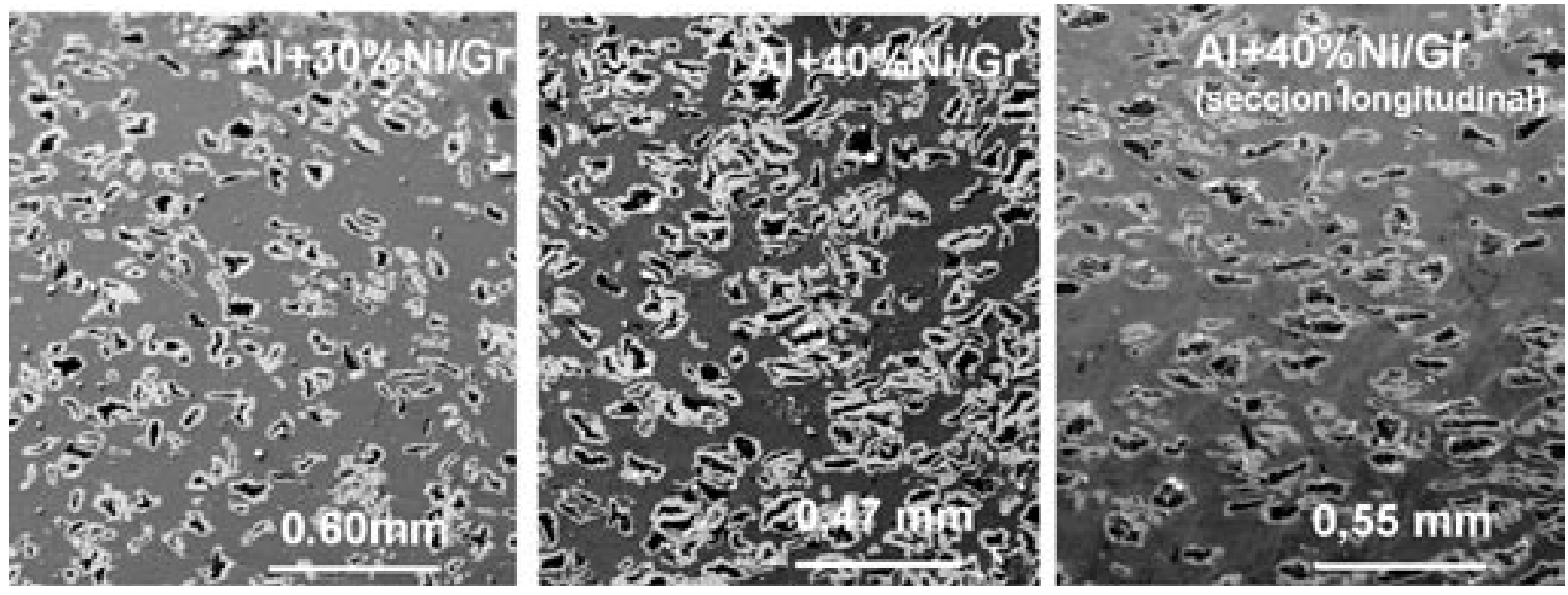

Figura 1. Microestructura de los materiales extruidos.
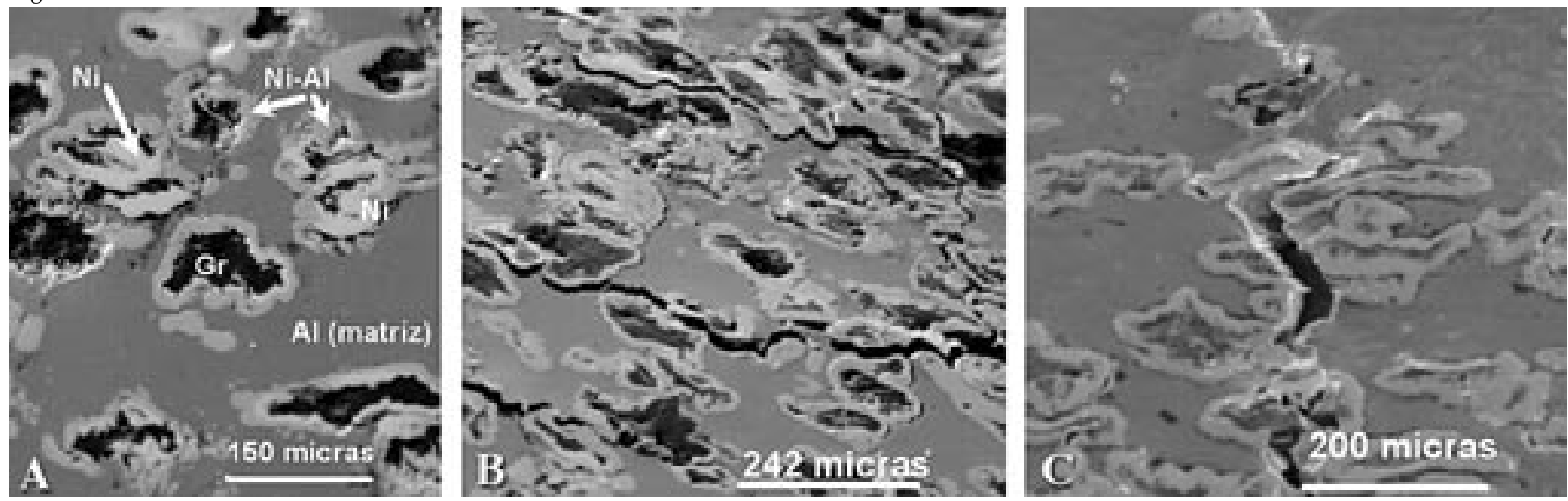

Figura 2. Microestructura del material extruido y tratado térmicamente $\mathrm{Al}+30 \% \mathrm{Ni} / \mathrm{Gr}(\mathrm{A})$ y Superficie de fractura a flexión de los materiales extruidos y tratados térmicamente (B y $\mathrm{C}$ ) 

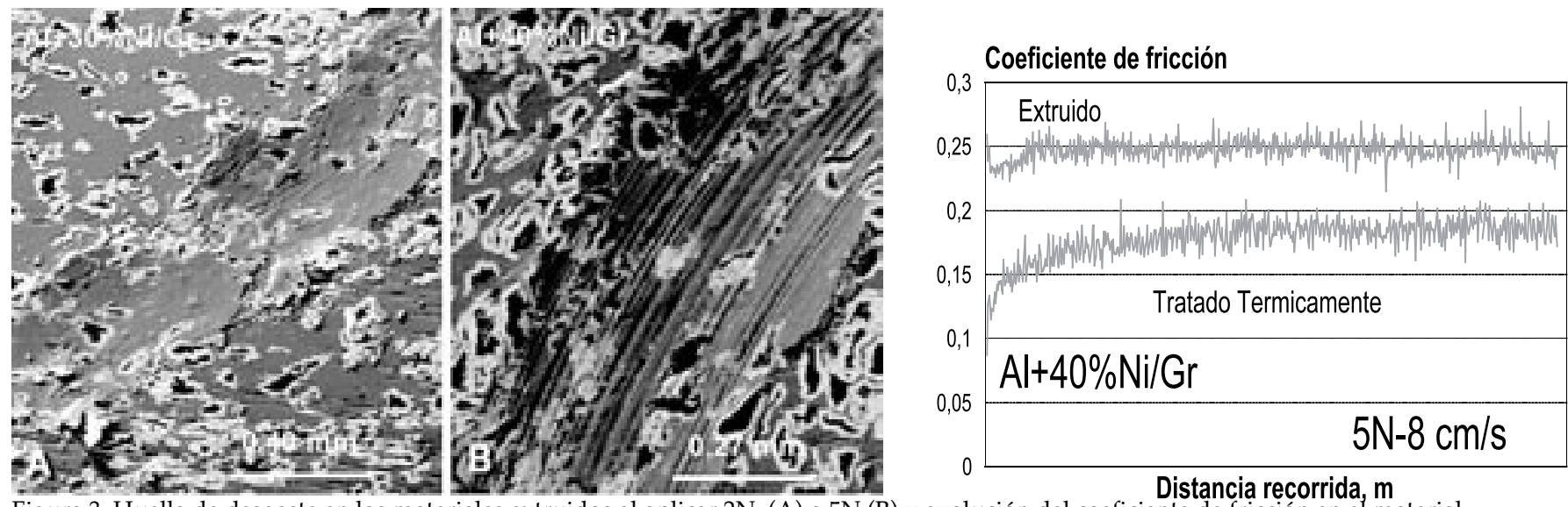

Figura 3. Huella de desgaste en los materiales extruidos al aplicar $2 \mathrm{~N}$ (A) o $5 \mathrm{~N}$ (B) y evolución del coeficiente de fricción en el material $\mathrm{Al}+40 \% \mathrm{Ni} / \mathrm{Gr}$

un cambio en la microdureza alcanzándose valores de 360-488 HV0.5N en estas áreas de $\mathrm{Ni}-\mathrm{Al}$, respecto de unas microdurezas de $40-50 \mathrm{HV} 0.5 \mathrm{~N}$ en la matriz de $\mathrm{Al}$ y de 60-91HV0.5N en las areas de níquel.

\subsection{Propiedades mecánicas.}

La dureza disminuye al aumentar el contenido en grafito, desde $57-67 \mathrm{HRH}$ para el $\mathrm{Al}+30 \% \mathrm{Ni} / \mathrm{Gr}$ a $47-58$ en el $\mathrm{Al}+40 \% \mathrm{Ni} / \mathrm{Gr}$. Tras el tratamiento térmico a $525^{\circ} \mathrm{C} / 4 \mathrm{~h}$, se alcanzaron unas durezas de 54 $65 \mathrm{HRH}$ y $47-53 \mathrm{HRH}$ respectivamente. La resistencia al aplastamiento radial también depende del contenido en grafito, alcanzándose 203$218 \mathrm{MPa}$ para el $\mathrm{Al}+30 \% \mathrm{Ni} / \mathrm{Gr}$ y de $165-176 \mathrm{MPa}$ para el $\mathrm{Al}+40 \% \mathrm{Ni} /$ $\mathrm{Gr}$, ambos dentro de los valores especificados para cojinetes de hierrocarbono-grafito[8]. Tras el tratamiento térmico, disminuye fuertemente la resistencia al aplastamiento radial al reducirse la tenacidad del material como consecuencia de la formación de Al-Ni y se obtiene resistencias de 126-132MPa y 49-59MPa respectivamente en ambos materiales.

El análisis de la fractura en los casquillos ensayados a rotura y en las probetas ensayadas a flexión, muestran que si en el material extruido una parte importante de las grietas atraviesan la partícula de $\mathrm{Ni}-\mathrm{Gr}$, este mecanismo no se produce después del tratamiento térmico, rodeando las áreas de $\mathrm{Ni}-\mathrm{Al}$ y provocando el despegue de estas áreas de la matriz de aluminio (Figura 2B y C).

\subsection{Propiedades tribiológicas}

La adhesión del aluminio a la bola de acero y la abrasión de ésta sobre el material debido a la deformación plástica de la matriz, son los mecanismos de desgaste observados en estos materiales [7]. La presencia de grafito en el material conduce a que se adhiera a la bola disminuyendo la facilidad de adhesión del aluminio a ésta. Mayores cargas de ensayo ( $5 \mathrm{~N}$ en lugar de $2 \mathrm{~N}$ ) favorecen una mayor incorporación del grafito a la pista de desgaste y se alcanza un menor grado de adhesión y por ello una menor fluctuación del coeficiente de fricción, asi como éste disminuye después del tratamiento térmico (Figura 3). Respecto a la deformación plástica experimentada por la matriz de aluminio, dado que éste no ve mejorada su dureza por la incorporación del níquel, sólo la formación de las áreas de Ni-Al en las partículas iniciales de $\mathrm{Ni} / \mathrm{Gr}$ conduce a que predomine un mecanismo de abrasión por microgrietas, por el material arrancado o por corte, que por el de deformación plástica de la matriz. La medición mediante un perfilómetro de las huellas generadas condujo a que el ancho de la pista generada aumentaba cuando lo hacia la carga aplicada $(2 \mathrm{~N}$ o $5 \mathrm{~N})$, menor es el contenido en grafito y en estado de extrusión (Figura $3 \mathrm{Ay}$
B). Por ello el material que mejor comportamiento a desgaste presentó fue el $\mathrm{Al}+40 \% \mathrm{Ni} / \mathrm{Gr}$ una vez tratado térmicamente .

\section{CONCLUSIONES}

Mediante la extrusión de polvos se puede obtener materiales compuestos de matriz de aluminio con una adecuada distribución de grafito rodeado por el níquel. Este grafito contribuye a disminuir el coeficiente de fricción de estos materiales mejorando sus propiedades tribológicas, especialmente al reducir los mecanismos de adhesión del aluminio a la bola de acero. Mediante un tratamiento térmico se mejoran sus propiedades tribológicas, si bien se disminuye su resistencia al aplastamiento radial, resultando el desgaste de estos materiales debido, fundamentalmente, a mecanismos de abrasión. La incorporación de partículas de refuerzo a la matriz de aluminio se plantea como un solución para mejorar aún más la resistencia al desgaste de estos materiales.

\section{AGRADECIMIENTOS}

Deseamos agradecer a la empresa Novamet por el suministro de los polvos de níquel-grafito.

\section{BIBLIOGRAFIA}

1. LEG Cambronero et al. "Radial crushing strength of bronze with nickel-graphite additions". Euromat 99, vol 8, Munich, 315-322, (1999)

2. LEG Cambronero et al."Characterization of nickel bronze graphite composites for friction applications". PM98 Word Congress, Granada 18-22 October, Spain, Vol 74, 349-354, (1998)

3. S.F. Moustafa et al."Effect of graphite with and without copper coating on consolidation behaviour and sintering of copper-graphite composite". Pow. Met., vol 40, 3, 201-205, (1997)

4. H Chung et al. "The effect of graphite on the processing and properties of copper-graphite composite". Adv in PM, June 16-21, Orlando USA, Vol 6 117-128, (2002).

5. AEM Warner. "Opportunities for new graphitic aluminium metal matrix composite". Materials Science and Technology, Vol 14, 843-850, (1998)

6. A.R. Riahi, A.T. Alpas ."The role of tribo-layers on the sliding wear behaviour of graphitic aluminium matrix composites". Wear 251, 1396-1407, (2001)

7. LEG Cambronero et al ."Mechanical characterization of AA7015 aluminium alloy reinforced with ceramics". AMPT02, Leganes, Spain, September 18-21, 1513-1520, (2002)

8. ISO 5755. Sintered metal materials-Specifications. (2001)

Recibido: 1.2 .03

Aceptado: 30.11 .03 